\title{
Changing form and function during development in rowing hydromedusae
}

\author{
Jacqueline Weston ${ }^{1}$, Sean P. Colin ${ }^{1, *}$, John H. Costello ${ }^{2}$, Emily Abbott ${ }^{2}$ \\ ${ }^{1}$ Environmental Science/Marine Biology, Roger Williams University, Bristol, Rhode Island 02908, USA \\ ${ }^{2}$ Biology Department, Providence College, Providence, Rhode Island 02918, USA
}

\begin{abstract}
Bell morphology, propulsion and foraging appear to be interdependent traits among medusan lineages. In general, taxa that possess large oblate bells swim via rowing propulsion and forage as cruising predators, and taxa with small prolate bells swim via jet propulsion and forage as ambush predators. However, hydromedusae from the taxa Leptothecata (referred to here as leptomedusae) experience large changes in bell size and shape during their development. We used video analysis to evaluate changes in bell morphology and kinematics, fluid interactions and behavior of 2 leptomedusan species, Aequorea victoria and Eutonina indicans, during these developmental transitions. Both species begin as small, prolate, jetting juveniles ( $<1 \mathrm{~mm}$ diameter) and develop into large, oblate, rowing adults ( $>0.5 \mathrm{~cm}$ diameter). In addition to morphological and propulsive alterations, we observed changes in behavior, whereby as jetters they rarely swam and as rowers they swam nearly continuously. These findings demonstrate that bell fineness, velar aperture ratio and bell kinematics interact to determine fluid interactions and thus determine propulsive mode.
\end{abstract}

KEY WORDS: Functional morphology $\cdot$ Foraging behavior $\cdot$ Propulsion mode $\cdot$ Swimming behavior • Hydromedusae

\section{INTRODUCTION}

Jellyfish are among the earliest metazoans to have evolved muscular tissues for locomotion (Valentine 2004). One consequence of their early origin within the animal kingdom is their relatively simple body plan and a correspondingly narrow array of morphological structures available for propulsion and feeding (Gladfelter 1972a,b, Costello et al. 2008). The limited nature of cnidarian muscular tissues has constrained medusan propulsion to essentially 2 forms: rowing- and jetpropelled medusae (Dabiri et al. 2007, Costello et al. 2008). Each of these 2 functional types shares a suite of propulsive, morphological and behavioral traits (Mills 1981, Madin 1988, Colin \& Costello 1996, 2002).

Jet-propelled medusae are typically ambush foragers and possess prolate shaped bells with highly constricted orifices (i.e. high velar aperture ratio; Dabiri et al. 2006). Swimming by jet-propelled medusae involves rapid bell contraction and forceful ejection of a fluid bolus from their subumbrellar cavity.
This process propels jetting medusae forward with relatively high acceleration rates. Despite their often highly effective swimming capacities, jet-propelled medusae are typically ambush-foraging predators that spend much of their time relatively motionless. They depend upon prey to swim into outstretched tentacles (Colin \& Costello 2002). In contrast, rowing-propelled medusae are typically cruising foragers and have more oblate bells and less constricted orifices, and they swim almost continuously via rhythmic bell contractions (Colin \& Costello 2002, Dabiri et al. 2005, 2006). Wake dynamics of rowing species tend to be more complex than those of their jet-propelled counterparts (Dabiri et al. 2005, 2007). While not as rapid swimmers, rowing medusae swim more efficiently, and entrain more fluid in their wakes than jetting medusae. These 2 traits make rowing propulsion suitable for medusae which forage as cruising predators (Dabiri et al. 2005).

The distribution of propulsive strategies among medusan species is broadly related to medusan size. Prolate, jetting medusae are generally limited to an 
upper size range of roughly 5 to $10 \mathrm{~cm}$ in bell diameter (Dabiri et al. 2007, Costello et al. 2008). Above that size threshold, medusae are not capable of exerting sufficient muscular force for jet propulsion. As a result, most prolate medusae are relatively small. This same constraint does not exist for rowing oblate medusae because they do not need to exert as much force with their bells to move (Dabiri et al. 2007, Costello et al. 2008). Consequently, oblate medusae are capable of body dimensions greater than $1 \mathrm{~m}$ in bell diameter (e.g. Kawahara et al. 2006).

This relationship between morphology and foraging mode has provided plankton ecologists a tool for evaluating the ecological roles of a range of medusan species. Medusae are generally evaluated based on adult traits (e.g. Larson 1987, Purcell \& Mills 1988, Costello \& Colin 2002, Sötje et al. 2007). However, some medusan taxa, particularly hydromedusae from the taxa Leptothecata (referred to hereafter as leptomedusae), develop from small, prolate juveniles to large, oblate adults (see Fig. 1A). Based on the broad relationships between morphological and functional traits, we would expect the changes in size and shape to be accompanied by alterations in propulsive and foraging traits. Further, based on the constraints upon force production by medusan subumbrellar muscular tissues, we might expect that the transition from small, prolate, jetting juveniles to large, oblate, rowing adults by leptomedusae would occur at sizes below the size constraint predicted by Dabiri et al. (2007).

We chose 2 leptomedusae whose entire life cycle is in culture at the New England Aquarium, Boston, Massachusetts, USA, in order to quantify alterations in form and function during leptomedusan development. We used video analyses to examine the swimming of the leptomedusae Aequorea victoria and Eutonina indicans through a range of developmental stages to detail alterations in bell morphology and kinematics, swimming performance, fluid interactions and behavior. These 2 species are common in Pacific northwest waters (Arai \& Brinckmann-Voss 1980), but other species of these genera are found throughout the world's oceans (Kramp 1961, Bouillon et al. 2004).

\section{MATERIALS AND METHODS}

Experimental organisms. We had access to 2 leptomedusan species, Aequorea victoria (Murbach \& Shearer 1902) and Eutonina indicans (Romanes 1876), that were cultured and generously supplied by the New England Aquarium, Boston, Massachusetts, USA. The aquarium provided us with medusae of all developmental stages. Once in the laboratory, medusae were maintained in 11 glass containers at a density of 8 to 10 individuals per container. The containers were placed on rolling platforms (1 rpm) and kept at a constant temperature of $13^{\circ} \mathrm{C}$ in a diurnal light cycle. This setup was an effective way to maintain the medusae for weeks by keeping them suspended and preventing them from aggregating on the bottom of vessels where bacteria can deteriorate the tentacles and bells of medusae.

Video recording. To visualize the morphology and kinematics of swimming medusae and fluid around their bells, individuals were placed in $64 \mu \mathrm{m}$ filtered seawater in glass vessels with dimensions ranging from $4.0 \times 8.0 \times 1.0 \mathrm{~cm}$ to $25.2 \times 30.4 \times 18.4 \mathrm{~cm}$ (width $\times$ height $\times$ depth). The size of the vessel depended upon the size of the medusae, using vessels which allowed an individual to swim freely while still minimizing the depth of field.

Each individual was filmed freely swimming for approximately 15 to 20 min using a backlit optical system (Costello \& Colin 1994) and either a standard rate/standard definition Pulnix charge-coupled device camera (30 frames $\mathrm{s}^{-1} / 480 \times 640$ pixel resolution) or a high speed/standard definition Fastcam Super 10K high speed video system (Photron; 250 to 500 frames $\mathrm{s}^{-1} / 480 \times 640$ pixel resolution). All video was recorded onto mini-DV tape and analyzed using Adobe Premiere Pro and ImageJ (developed by the National Institute of Health, NIH) software. To examine the flow around the bell of swimming medusae, fluorescein dye was injected into the fluid adjacent to the bell using a repeat pipettor at $5 \mu$ aliquots (Dabiri et al. 2005).

Video analysis. Bell morphology data were collected from images in which the medusa was maximally relaxed and the ventral surface was oriented perpendicular to the camera. Bell fineness $(F)$ (Daniel 1983, Costello \& Colin 1994, Colin \& Costello 2002, Dabiri et al. 2007), which defined bell shape and indicated progress through the bell contraction cycle, was calculated using:

$$
F=\frac{h}{d}
$$

where $h$ is bell height and $d$ is bell diameter. The velar aperture was measured from medusae at rest because of the difficulty of imaging the velum during bell contraction. $d$ and the diameter of the velar aperture $(v)$ were measured from each of the medusae videotaped. Assuming that the velar aperture and bell were circular in cross-section, the ratio of their areas, the velar aperture area ratio $\left(R_{\mathrm{v}}\right)$, can be simplified to a percentage based on the relationship:

$$
R_{\mathrm{v}}=\frac{v^{2}}{d^{2}}
$$

Bell kinematics were quantified using the contraction time of the bell (i.e. time elapsed from maximum 
expansion to maximum contraction) and the time between pulses (i.e. amount of time the bell remained in maximum expansion during bell pulsation).

Both bell morphology and kinematics determine how medusae interact with their surrounding fluids during swimming. The prolate bells, narrow apertures and rapid contractions of jetting species result in medusae expelling a single starting vortex during the pulsation cycle that travels rapidly away from the medusa in the trailing wake (Dabiri et al. 2006). The oblate bells, broad apertures and slow contraction rates of rowing species result in medusae creating 2 vortex rings (the starting and stopping vortices), with opposite rotation during each pulsation cycle. The interaction of these vortices reduces the total amount of momentum imparted to the surrounding fluid and the rate at which the vortices travel away from the medusae in the trailing wake (Dabiri et al. 2005, 2007). These different wake structures define and distinguish each propulsion mode (Dabiri et al. 2005, 2006). There-

A

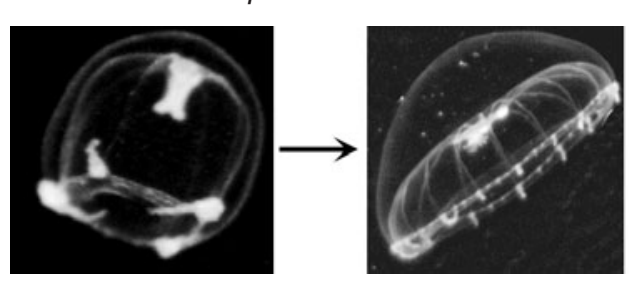

B

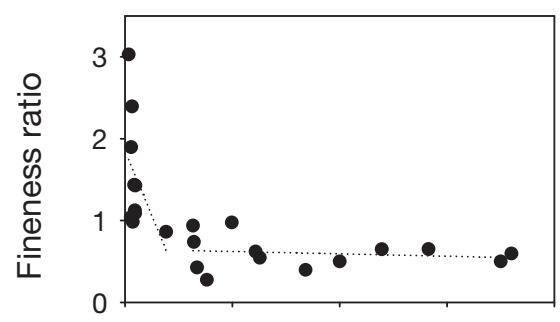

C

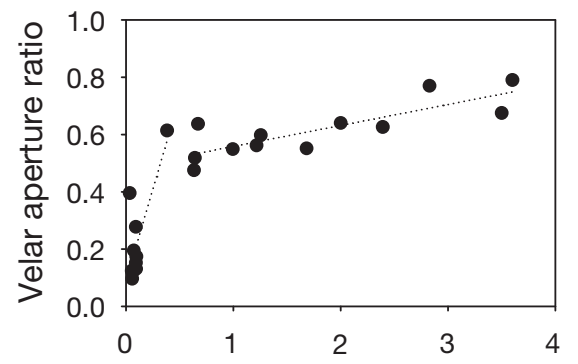

fore, we measured the velocity of the trailing vortex rings in the wake of the different medusae during bell contraction to help distinguish the type of propulsion used by Aequorea victoria and Eutonina indicans throughout their development. The velocity of a vortex ring was measured as the distance that the center of the ring traveled during bell contraction and divided by contraction time.

To measure swimming kinematics, we used sequences in which the medusae swam upwards in the viewing plane and perpendicular to the viewing direction for several pulsation cycles. The video sequences were separated into $0.02 \mathrm{~s}$ intervals. The change in positions $(x, y)$ of the medusae (using the bell apex as the reference point) were tracked over time $(t)$ and used to calculate velocity $(u)$ throughout the pulsation cycle as:

$$
u=\frac{\sqrt{\left(x_{t+1}-x_{t}\right)^{2}+\left(y_{t+1}-y_{t}\right)^{2}}}{\Delta t}
$$

\section{Eutonina indicans}
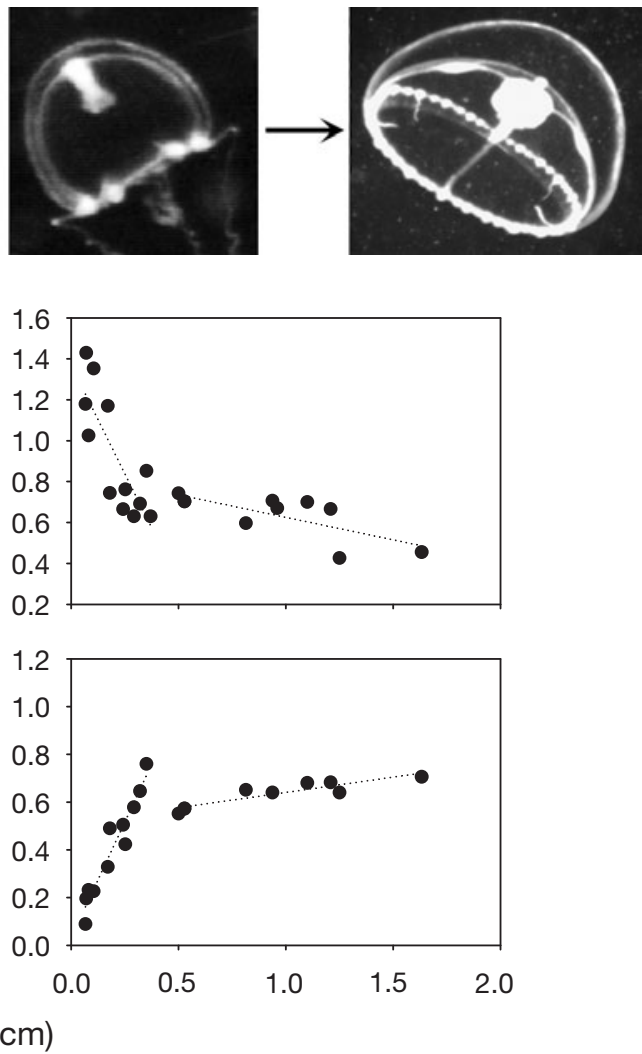

Fig. 1. Aequorea victoria and Eutonina indicans. (A) Prolate (left panels) and oblate (right panels) forms of each species, (B) fineness ratio (bell height/diameter) and $(\mathrm{C})$ velar aperture ratio $\left(R_{\mathrm{v}}=\right.$ velar diameter $\left.{ }^{2} / \mathrm{bell}^{\text {diameter }}{ }^{2}\right)$ of $A$. victoria and $E$. indicans medusae at different stages of development. Fineness decreases rapidly with diameter up to $5 \mathrm{~mm}$ (linear regression of individuals $<0.5 \mathrm{~mm}$ for $A$. victoria: $y=1.86-3.18 x, \mathrm{r}^{2}=0.20, \mathrm{p}<0.01$; and E. indicans: $y=1.37-2.12 x, \mathrm{r}^{2}=0.66$, $\mathrm{p}<0.01$ ) then decreases more slowly ( $>0.5 \mathrm{~mm}$ for A. victoria: $y=0.65-0.023 x, \mathrm{r}^{2}=0.020, \mathrm{p}>0.1$; and E. indicans: $y=0.84-0.22 x, \mathrm{r}^{2}=0.53, \mathrm{p}<0.02$ ). Velar aperture rapidly increases with diameter up to $5 \mathrm{~mm}\left(<0.5 \mathrm{~mm}\right.$ for $A$. victoria: $y=0.09+1.28 x, \mathrm{r}^{2}=0.60, \mathrm{p}<0.01$; and $E$. indicans: $\left.y=0.03+1.93 x, r^{2}=0.92, p<0.01\right)$ then continues more slowly $\left(>0.5 \mathrm{~mm}\right.$ for A. victoria: $y=0.48+0.073 x, \mathrm{r}^{2}=0.79$, $\mathrm{p}<0.01$ and E. indicans: $\left.y=0.51+0.128 x, \mathrm{r}^{2}=0.83, \mathrm{p}<0.01\right)$ 


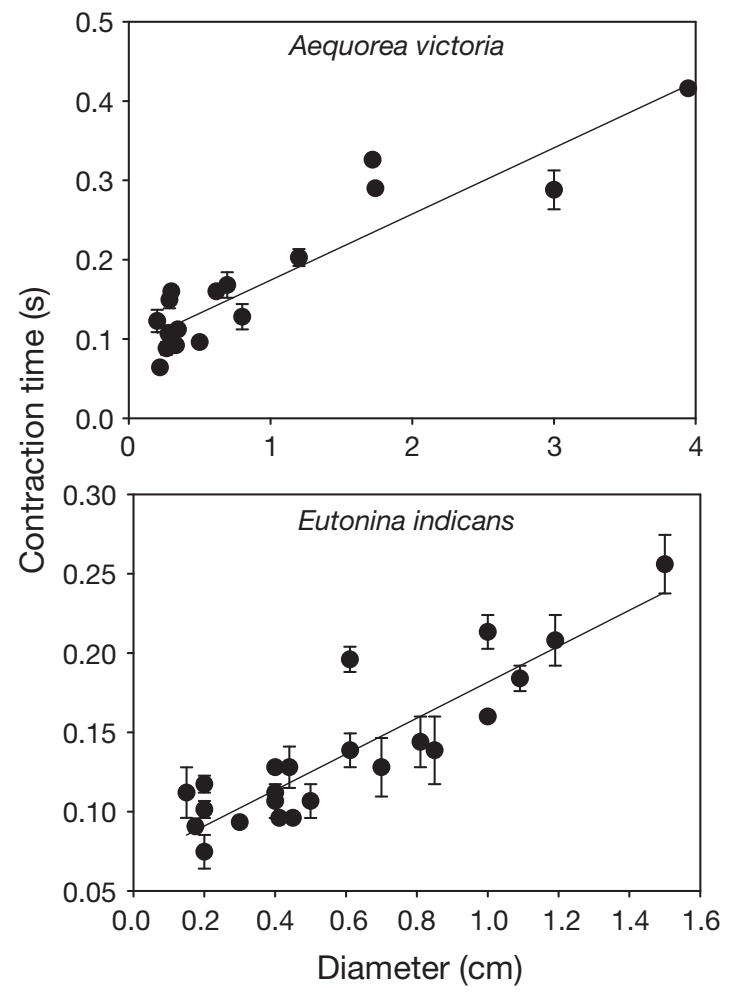

Fig. 2. Aequorea victoria and Eutonina indicans. Duration of bell contraction during the pulsation cycle at different stages of development. Each data point is the average $( \pm \mathrm{SD})$ of 2 to 4 consecutive contractions by a swimming medusa. Increase with size was linear (linear regression $\mathrm{p}<0.001$; A. victoria: slope $=0.083, \mathrm{r}^{2}=0.87 ;$ E. indicans: slope $=0.11, \mathrm{r}^{2}=0.72$ )

where $x$ and $y$ correspond to the $x$ - and $y$-coordinates of the bell apex.

Behavior was quantified by continuously videotaping individuals for $15 \mathrm{~min}$ and measuring the proportion of time spent swimming versus resting with tentacles extended. It has been shown that cruising medusae swim nearly continuously with their tentacles extended (Mills 1981, Costello et al. 1998, Colin et al. 2003), while ambush-foraging medusae swim only occasionally and rest a large majority of their time (Mills 1981, Colin et al. 2003). In situ observations have shown that both Eutonina indicans and Aequorea victoria exhibit cruising behavior as adults, swimming nearly continuously with their tentacles extended (Colin et al. 2003).

\section{RESULTS}

The bell morphology of Aequorea victoria and Eutonina indicans changed rapidly during the early stages of development (Fig. 1). Newly budded medusae of both species were very small $(<1 \mathrm{~mm}$ diameter) and highly prolate, with bell lengths greater than bell widths $(F<1$; Fig. 1B). By $2 \mathrm{~mm}$ in diameter, the bells of both species were wider than long $(F>1)$ and by $4 \mathrm{~mm}$ in diameter medusae had oblate shaped bells with similar fineness ratios to adults. Concurrently, as medusae grew, their orifices became less constricted (Fig. 1C). For both species, these morphological changes occurred early in development. By $5 \mathrm{~mm}$ in diameter, their bells changed from being prolate with highly constricted orifices to oblate with minimally constricted orifices. After $5 \mathrm{~mm}$, the bells continued to become more oblate and less constricted but the change occurred more slowly (Fig. 1).

Along with bell morphology, bell kinematics during swimming changed early in development. Contraction times increased during development (Fig. 2) and the time between consecutive pulses decreased (Fig. 3).

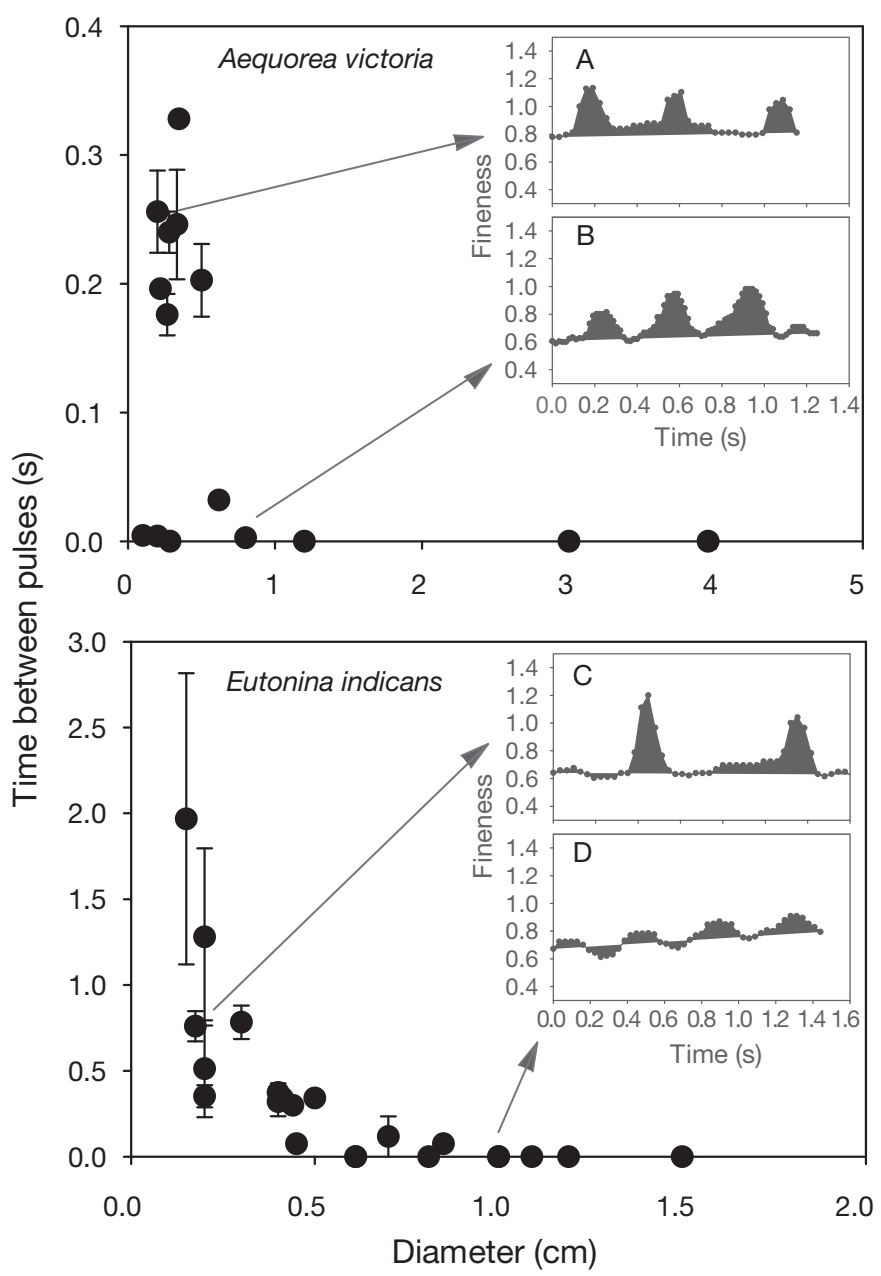

Fig. 3. Aequorea victoria and Eutonina indicans. Time between consecutive pulses at different stages of development. Each data point is the average $( \pm \mathrm{SD})$ of 2 to 4 consecutive swimming cycles of each individual. Arrows point to sample fineness ratio versus time swimming profiles of $(A, C)$ small and $(\mathrm{B}, \mathrm{D})$ large individuals of each species. Peaks in fineness represent points of maximum contraction in a swimming cycle 

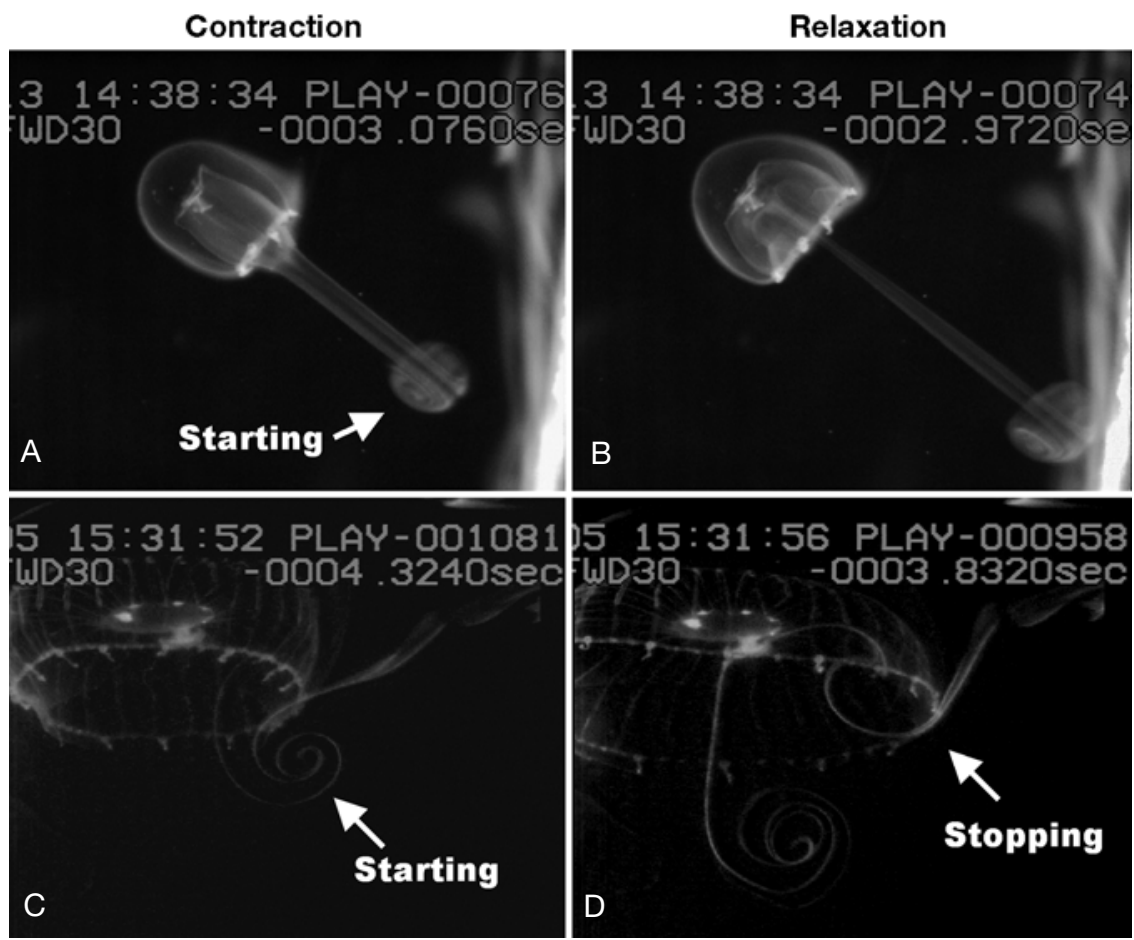

Fig. 4. Aequorea victoria. Wake visualization of swimming A. victoria at $(\mathrm{A}, \mathrm{B}) 2 \mathrm{~mm}$ and $(\mathrm{C}, \mathrm{D}) 10 \mathrm{~mm}$ diameter during the contraction and relaxation phases of the swimming cycle. Arrows point to the starting vortex during the contraction phase and stopping vortex during the relaxation phase. No stopping vortex is formed during the relaxation phase of the $2 \mathrm{~mm}$ medusae

The smallest medusae of both species contracted their bells in $<0.1 \mathrm{~s}$, and then bell contraction times increased linearly with diameter. In addition to short contraction times, the smallest medusae swam with pauses between each bell pulsation (Fig. 3A,C), while larger individuals swam with continuous pulsation cycles (Fig. 3B,D). The duration of the pauses observed among small prolate medusae was highly variable; in contrast, those of large medusae were highly consistent because none of the larger oblate individuals paused between pulsation cycles. Again, this transition occurred by the size of approximately $5 \mathrm{~mm}$ in bell diameter.

As a result of these morphological and kinematic transitions, the wake structure of the medusae changed with size. As is characteristic of jet propulsion, small, prolate medusae created only starting vortex rings (Fig. 4) which rapidly traveled away from the medusae in the trailing wake (Fig. 5). As the medusae grew, stopping vortices were also observed (Fig. 4). As

Fig. 5. Aequorea victoria and Eutonina indicans. Velocity of trailing vortex rings as they travel away from swimming medusae at different stages of development. Each data point is the average $( \pm \mathrm{SD})$ of 2 to 4 velocities observed over consecutive swimming cycles of each individual

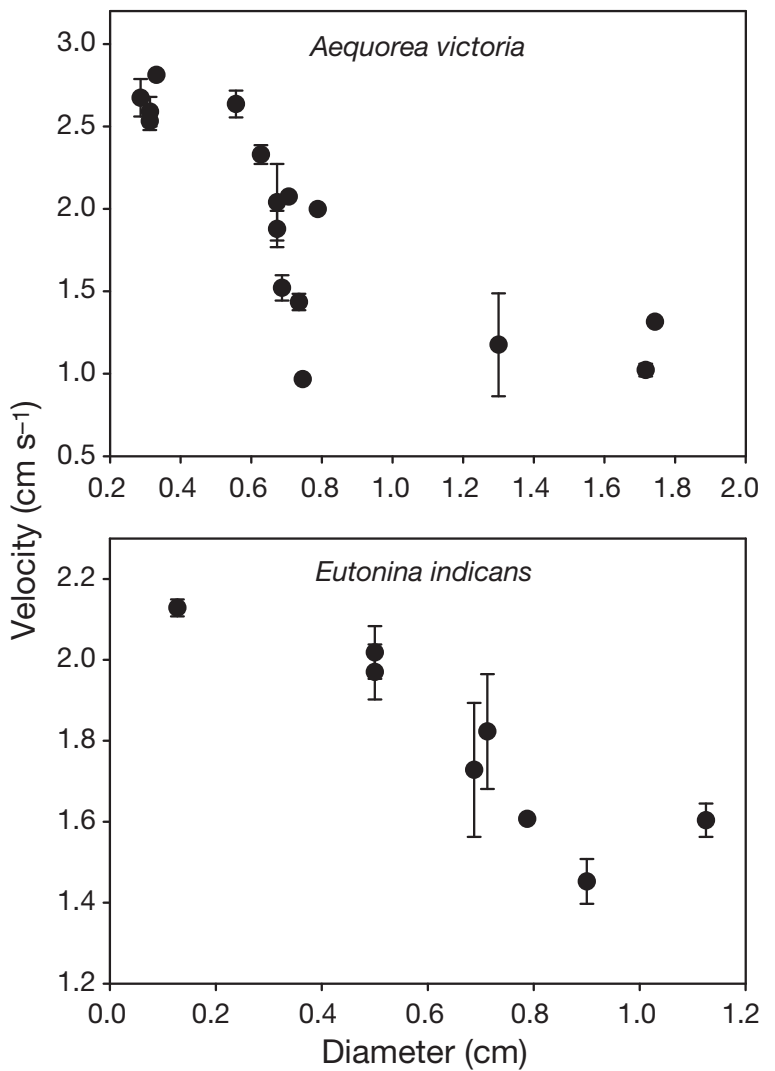




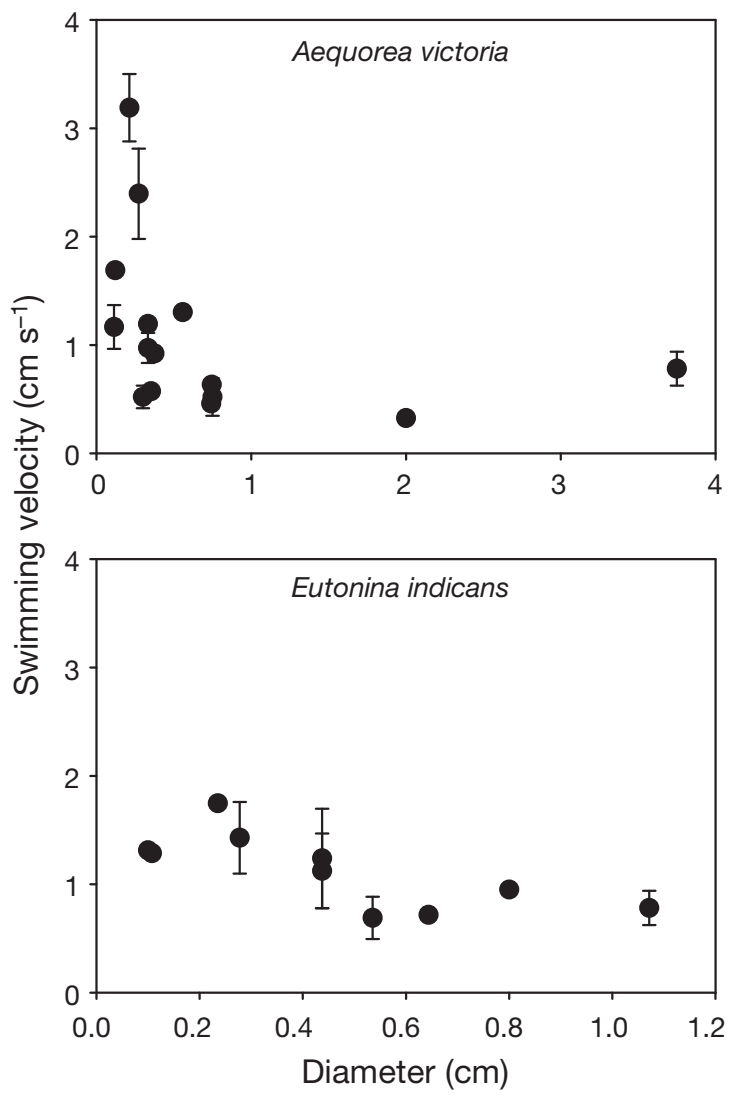

Fig. 6. Aequorea victoria and Eutonina indicans. Peak swimming velocity at different stages of development. Each data point is the average $( \pm \mathrm{SD})$ of 2 to 4 maximum velocities observed over consecutive swimming cycles of each individual

is characteristic of rowing propulsion, the starting and stopping vortices of the large medusae interacted to slow the velocity with which trailing vortices traveled away from the medusae (Fig. 5). Consistent with this transition from jetting to rowing, the swimming velocity of both Aequorea victoria and Eutonina indicans decreased as the medusae developed (Fig. 6). It is important to note that these decreases in swimming velocity with increasing bell diameter were observed during development of both species even as the medusae increased bell diameter by more than an order of magnitude.

Behavioral changes accompanied morphological and kinematic development. For both species, the smallest individuals rarely swam and larger individuals swam nearly continuously (Fig. 7). The transition occurred by 0.8 and $0.6 \mathrm{~cm}$ bell diameter for Aequorea victoria and Eutonina indicans, respectively. The transition in swimming behavior occurred over relatively narrow bell diameter ranges (Fig. 7) and few individuals of either species were observed swimming for intermediate proportions of time.

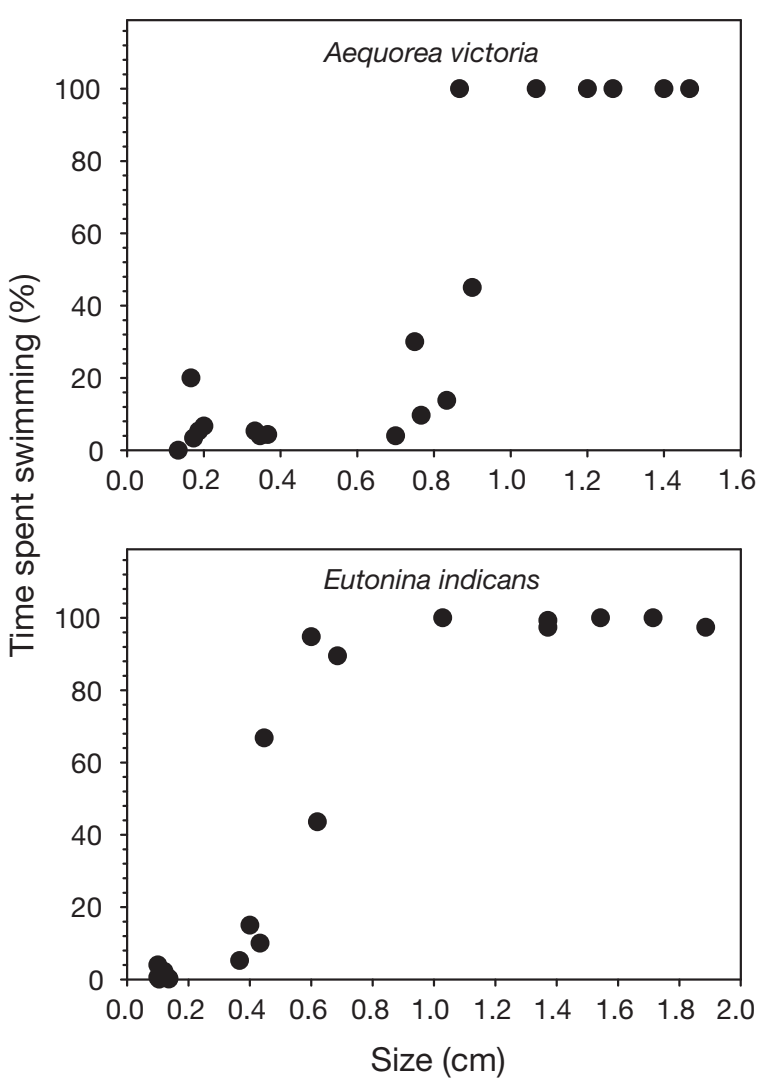

Fig. 7. Aequorea victoria and Eutonina indicans. Percentage of time medusae at different stages of development were observed swimming over a 15 min observation period

\section{DISCUSSION}

\section{Determinates of propulsive mode}

Bell morphometrics and swimming kinematics provide insight into the major traits associated with either propulsive mode. Jet propulsion is enhanced by small velar orifices and rapid bell contractions, which increase jet thrust and minimize form drag of prolate bells (Daniel 1983, 1985, Colin \& Costello 1996, 2002, Dabiri et al. 2006). Less is known about characteristics which maximize the efficiency of rowing propulsion. However, the interaction of the stopping and starting vortices is crucial for rowing propulsion. This interaction increases fluid entrainment and swimming efficiency while it decreases the force generation requirements of the medusa (Dabiri et al. 2005, 2007). As the fineness ratio decreases (i.e. medusae become more oblate), the size of the stopping vortex increases and the advantages of rowing propulsion are enhanced (e.g. swimming efficiency and fluid entrainment; Dabiri et al. 2005, 2007). Our observations of Aequorea victo- 
ria and Eutonina indicans demonstrate that reduced velum dimensions and continuous pulsation cycles may also be important for rowing propulsion because they enable interactions between the starting and stopping vortices of the medusan wake. The stopping vortex is formed during the relaxation phase of bell pulsation and the starting vortex during the contraction phase of the next pulse. The stopping vortex is primarily contained within the subumbrellar cavity, while the starting vortex is located solely in the wake (Fig. 4). A large velum separates these 2 regions and obstructs interactions between the vortices. A small (as in oblate hydromedusae) or absent (as in scyphomedusae) velum allows the vortices to interact spatially by minimizing any physical obstruction between the vortices caused by the medusa's body. Further, a reduced velum enables the stopping vortex to exceed the subumbrellar cavity dimensions, thereby increasing its influence on the subsequent starting vortex (Dabiri et al. 2007). For the vortices to interact, they must also coincide in time: the starting vortex must form before the stopping vortex stops circulating. The continuous pulsation cycles of a large medusa with a reduced velum allows formation of a starting vortex while the previous stopping vortex is still circulating near the bell margin. Hence, small velar dimensions allow spatial proximity while continuous pulsation allows temporal proximity of starting and stopping vortices.

\section{Changes in form and function}

Developmental changes in the morphology of Aequorea victoria and Eutonina indicans were accompanied by changes in function that were consistent with predictions based on the observed relationship between form and function among medusan species (Costello et al. 2008). Specifically, both species developed from small, prolate, jetting medusae to large, oblate, rowing medusae, a transition which occurred below the critical size limit for jet-propelled medusae based on models of force-balance relationships (Dabiri et al. 2007, Costello et al. 2008). Behavioral patterns altered in synchrony with morphological development: individuals rarely swam as small, prolate jetters and swam nearly continuously as larger, oblate rowers. These behavioral changes suggest that the medusae may also be changing their foraging modes from ambush to cruising predation. Such a transition would be consistent with the observed foraging behavior of adult E. indicans, A. victoria and other leptomedusae in the field (Colin et al. 2003). However, we cannot say for certain that the different stages are foraging as ambush or cruising predators without observations of their feeding behavior or in situ swimming time budgets. In fact, it has been noted that rowing medusae are capable of foraging with both strategies (Colin et al. 2003) and other species of Aequorea appear to forage as ambush-foraging predators (A. vitrina, Riisgård 2007).

The functional transition between propulsive modes was rapid and occurred early in development. The relatively early transitions may not be determined by physiological limits because the transitions occurred at sizes well below physiological limits to jetting calculated by propulsive models (Dabiri et al. 2007). Perhaps there are ecological advantages to an early transition, e.g. as adults, cruising medusae are known to have greater ingestion rates than ambush-foraging predators (Matsakis \& Conover 1991). If juvenile cruising species also have higher ingestion rates, an early transition may provide growth advantages. Reasons for the relatively narrow size range over which the transitions occur also remain unclear. However, these patterns may indicate that there are disadvantages to using a hybrid mode of propulsion. The potentially disadvantageous nature of intermediate propulsive modes has been suggested as a reason for the conspicuous absence of hybrid-propulsive mode medusae among adults across a broad range of medusan taxa (Costello et al. 2008).

\section{CONCLUSION}

The morphological and functional changes that occur during the development of the leptomedusae Aequorea victoria and Eutonina indicans emphasize the strong interrelationships between morphology, propulsion and behavior in medusae. This strong relationship might be expected, since propulsive mode is determined by how the bell interacts with the surrounding fluid. Fineness ratio, velar aperture ratio and bell kinematics are all traits that determine fluid interactions and, consequently, fineness ratio alone is not a sufficient indicator of propulsion and foraging.

Other medusae such as Mitrocoma cellularia (Widmer 2004) and Craspedacusta sowerbyi (Boulenger \& Flower 1928) undergo similar morphological alterations during their development and forage as cruising predators as adults (Colin \& Costello 2002, Colin et al. 2006). Consequently, we believe the functional transitions observed here are likely to be common among a variety of medusae, particularly leptomedusae. Our results demonstrate that medusae require relatively few morphological and kinematic alterations to shift between propulsive modes. The relative ease of these transitions may have contributed to convergence upon either propulsive mode in several distantly related medusan lineages (Costello et al. 2008). 
Acknowledgements. We are grateful for the support of the National Science Foundation (OCE-0351398 and -0623534 awarded to S.P.C. and OCE-0350834, OCE 0623508 awarded to J.H.C.) and the Roger Williams University Research Foundation. We also thank S. Spina and the jellyfish crew at the New England Aquarium for their generous support with animals.

\section{LITERATURE CITED}

Arai M, Brinckmann-Voss A (1980) Hydromedusae of British Columbia and Puget Sound. Department of Fisheries and Oceans, Ottawa

Bouillon J, Medel MD, Pagès F, Gili JM, Boero F, Gravili C (2004) Fauna of the Mediterranean Hydrozoa. Sci Mar 68(Suppl 2):5-438

Boulenger MA, Flower WU (1928) The Regent's Park medusa, Craspedacusta sowerbyi, and its identity with C. (Microhydra) ryderi. Proc Zool Soc Lond 66:1005-1015

Colin SP, Costello JH (1996) Relationship between morphology and hydrodynamics during swimming by the hydromedusae Aequorea victoria and Aglantha digitale. Sci Mar 60:35-42

Colin SP, Costello JH (2002) Morphology, swimming performance and propulsive mode of six co-occurring hydromedusae. J Exp Biol 205:427-437

Colin SP, Costello JH, Klos E (2003) In situ swimming and feeding behavior of eight co-occurring hydromedusae. Mar Ecol Prog Ser 253:305-309

Colin SP, Costello JH, Kordula H (2006) Upstream foraging by medusae. Mar Ecol Prog Ser 327:143-155

Costello JH, Colin SP (1994) Morphology, fluid motion and predation by the scyphomedusa Aurelia aurita. Mar Biol 121:327-334

Costello JH, Colin SP (2002) Prey resource utilization by a guild of hydromedusae from Friday Harbor, Washington, USA. Limnol Oceanogr 47:934-942

> Costello JH, Klos E, Ford MD (1998) In situ time budgets of the scyphomedusae Aurelia aurita, Cyanea sp., and Chrysaora quinquecirrha. J Plankton Res 20:383-391

Costello JH, Colin SP, Dabiri JO (2008) Medusan morphospace: phylogenetic constraints, biomechanical solutions, and ecological consequences. Invertebr Biol 127:265-290

Dabiri JO, Colin SP, Costello JH, Gharib M (2005) Flow patterns generated by oblate medusan jellyfish: field measurements and laboratory analyses. J Exp Biol 208: $1257-1265$

Dabiri JO, Colin SP, Costello JH (2006) Fast-swimming jelly-

Editorial responsibility: Peter Verity,

Savannah, Georgia, USA fish exploit velar kinematics to form an optimal vortex wake. J Exp Biol 209:2025-2033

Dabiri JO, Colin SP, Costello JH (2007) Morphological diversity of medusan lineages constrained by animal-fluid interactions. J Exp Biol 210:1868-1873

Daniel TL (1983) Mechanics and energetics of medusan jet propulsion. Can J Zool 61:1406-1420

Daniel TL (1985) Cost of locomotion: unsteady medusan swimming. J Exp Biol 119:149-164

Gladfelter WB (1972a) Structure and function of the locomotory system of Polyorchis montereyensis (Cnidaria, Hydrozoa). Helgol Wiss Meeresunters 23:38-79

Gladfelter WB (1972b) Structure and function of the locomotory system of the Scyphomedusa Cyanea capillata. Mar Biol 14:150-160

Kawahara M, Uye S, Ohtsu K, Iizumi H (2006) Unusual population explosion of the giant jellyfish Nemopilema nomurai (Scyphozoa: Rhizostomeae) in East Asian waters. Mar Ecol Prog Ser 307:161-173

Kramp PL (1961) Synopsis of the medusae of the world. J Mar Biol Assoc UK 40:1-469

> Larson RJ (1987) Trophic ecology of planktonic gelatinous predators in Saanich Inlet, British Columbia: diets and prey selection. J Plankton Res 9:811-820

Madin LP (1988) Feeding behavior of tentaculate predators: in situ observations and a conceptual model. Bull Mar Sci 43:413-429

Matsakis S, Conover RJ (1991) Abundance and feeding of medusae and their potential impact as predators on other zooplankton in Bedford Basin (Nova Scotia, Canada) during spring. Can J Fish Aquat Sci 48:1419-1430

Mills CE (1981) Diversity of swimming behavior in hydromedusae as related to feeding and utilization of space. Mar Biol 64:185-189

Purcell JE, Mills CE (1988) The correlation between nematocyst types and diets in pelagic Hydrozoa. In: Hessinger DA, Lenhoff HM (eds) The biology of nematocysts. Academic Press, San Diego, CA, p 463-485

Riisgård HU (2007) Feeding behaviour of the hydromedusa Aequorea vitrina. Sci Mar 71:395-404

> Sötje I, Tiemann H, Båmstedt U (2007) Trophic ecology and the related functional morphology of the deepwater medusa Periphylla periphylla (Scyphozoa, Coronata). Mar Biol 150:329-343

Valentine JW (2004) On the origin of phyla. University of Chicago Press, Chicago, IL

Widmer CL (2004) The hydroid and early medusa stages of Mitrocoma cellularia (Hydrozoa, Mitrocomidae). Mar Biol 145:315-321

Submitted: June 9, 2008; Accepted: October 1, 2008 Proofs received from author(s): December 15, 2008 